\title{
Challenges and Pitfalls in the Management of Parathyroid Carcinoma: 17-Year Follow-Up of a Case and Review of the Literature
}

\author{
Janneke E. Witteveen • Harm R. Haak • Job Kievit • \\ Hans Morreau • Johannes A. Romijn • \\ Neveen A. T. Hamdy
}

Published online: 19 November 2010

(C) The Author(s) 2010. This article is published with open access at Springerlink.com

\begin{abstract}
A 29-year-old man presented to his primary care physician with nausea, severe weight loss and muscle weakness. He had a hard, fixed neck swelling. He was severely hypercalcaemic with 10 -fold increased parathyroid hormone (PTH) concentrations. A diagnosis of primary hyperparathyroidism was established and the patient was referred for parathyroidectomy. At neck exploration, an enlarged parathyroid gland with invasive growth into the thyroid gland was found and removed, lymph nodes were cleared and hemithyroidectomy was performed. A suspected diagnosis of parathyroid carcinoma was confirmed histologically. Serum calcium and PTH levels normalised post-operatively, but hyperparathyroidism recurred within 3 years of surgery. Over the following 17 years, control of hypercalcaemia represented the most difficult challenge despite variable success achieved with repeated surgical interventions, embolisations, radiofrequency ablation of metastases and treatment with calcimimetics, bisphosphonates
\end{abstract}

J. E. Witteveen · J. A. Romijn · N. A. T. Hamdy $(\bowtie)$

Department of Endocrinology \& Metabolic Diseases,

Leiden University Medical Center,

Albinusdreef 2,

2333 ZA, Leiden, The Netherlands

e-mail: N.A.T.Hamdy@LUMC.nl

H. R. Haak

Department of Internal Medicine, Máxima Medical Centre,

Eindhoven, The Netherlands

J. Kievit

Department of Surgery, Leiden University Medical Center, Leiden, The Netherlands

H. Morreau

Department of Pathology, Leiden University Medical Center, Leiden, The Netherlands and haemodialysis using low-dialysate calcium. In this paper, we report the challenges and pitfalls we encountered in the management of our patient over nearly two decades of followup and review recent literature on the topic.

Keywords Parathyroids · Parathyroidectomy.

Radiofrequency ablation - Embolisation - Cinacalcet .

HRPT2/CDC73

\section{Introduction}

Parathyroid carcinoma is a rare disorder, accounting for $0.1 \%$ to $5.0 \%$ of all cases of primary hyperparathyroidism [1-5]. The disease presents at a mean age of 50 years and is equally likely to occur in males as in females [2-6] (Table 1). Clinical and biochemical manifestations are those of severe primary hyperparathyroidism often with renal and skeletal complications [2-5, 7]. A palpable neck mass can be felt in 30-76\% of patients [3, 5, 8, 9]. Parathyroid carcinoma is associated with germline and somatic mutations in the HRPT2 gene $[3,10]$ and with irradiation of the neck for other pathologies $[3,11]$.

A diagnosis of parathyroid carcinoma is suggested by the presence of intra-operative features of local invasion and the diagnosis is confirmed by the World Health Organization histopathological criteria for parathyroid carcinoma. These include the presence of vascular invasion, perineural space invasion, capsular penetration with growth into adjacent tissues and/or metastasis [12].

Although parathyroid carcinoma is a slow-growing tumour, it inevitably metastasises and is invariably fatal due to the eventual inability to control hypercalcaemia. Survival is estimated to range from $50 \%$ to $86 \%$ at 5 years 


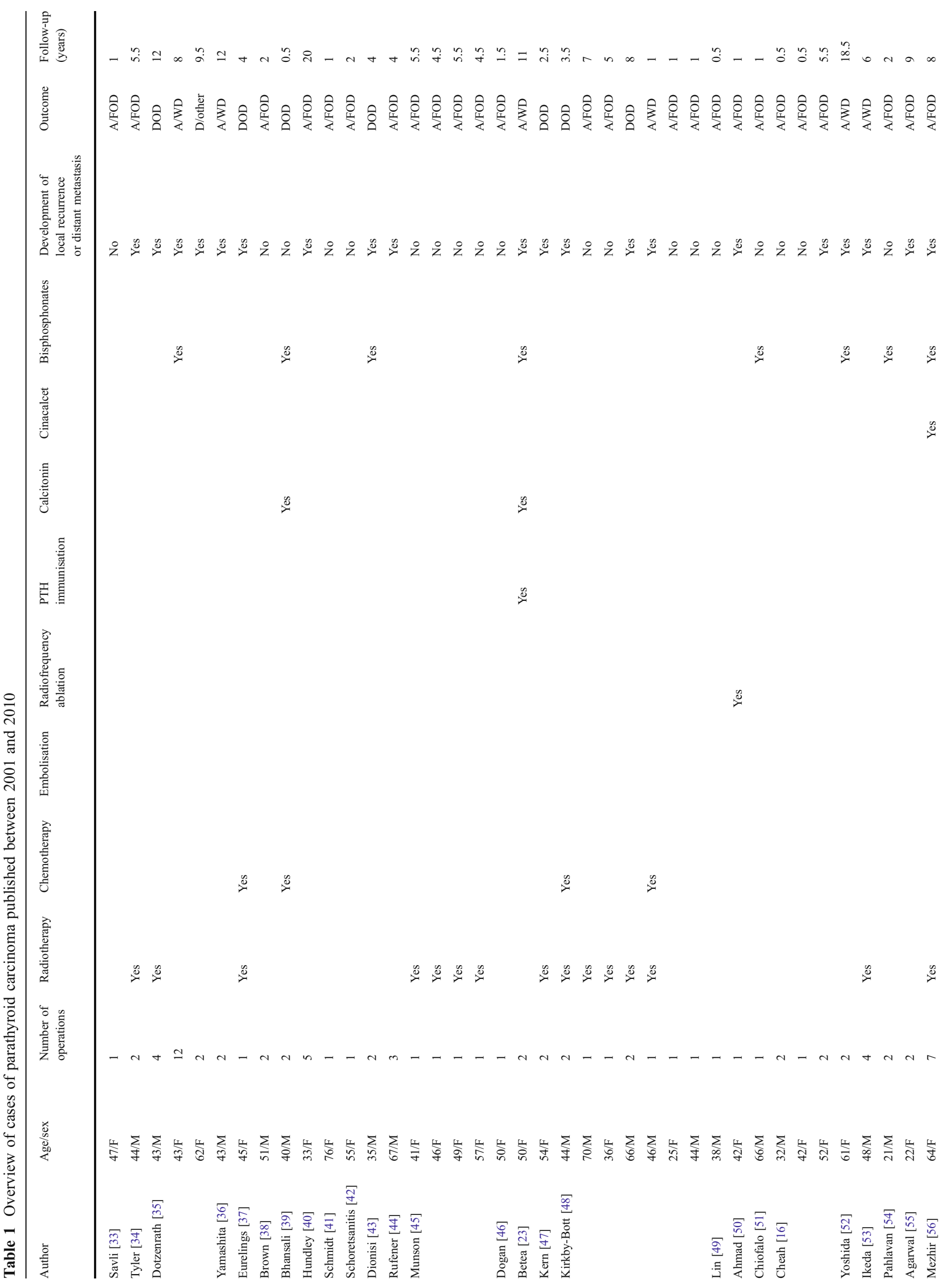




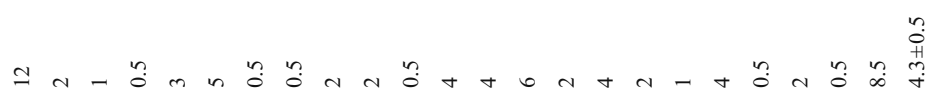

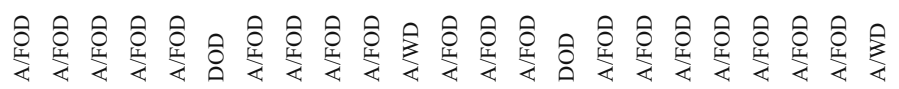

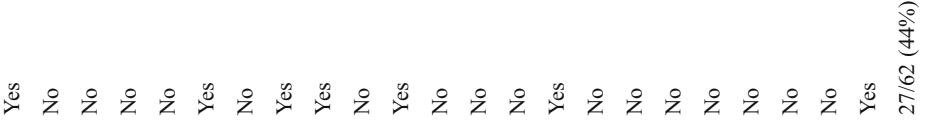

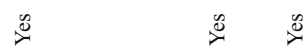

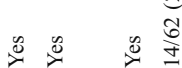

:

$\overbrace{2}^{\infty}$

$\frac{\sqrt{0}}{\frac{0}{2}}$

્ֻટ

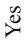

हैं

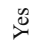

$\underbrace{\infty}$

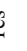

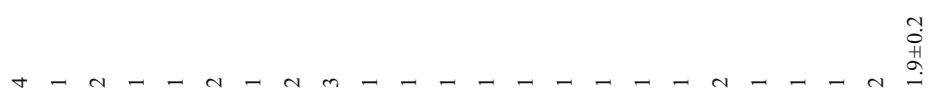

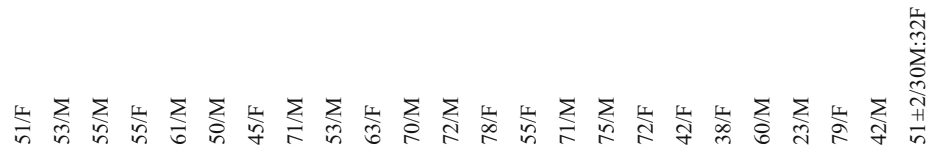

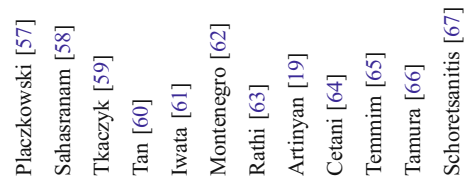


and $35-70 \%$ at 10 years $[1-6,13,14]$. The most effective treatment for parathyroid carcinoma is radical surgery with en bloc removal of the lesion together with the ipsilateral thyroid, thyroid isthmus and lymph nodes [2, 3, 5, 13, 15]. Surgery is also the treatment of choice in case of local recurrence or development of metastases [5, 7, 16], although radiotherapy $[2-5,8,9]$, chemotherapy $[3,5,9$, 17,18 ], embolisation [19] or radio frequency (RF) ablation [19-21] may also be attempted.

We report here the nearly two decades' odyssey of a male patient with multiple metastases from parathyroid carcinoma, describing in the process the challenges and pitfalls we encountered in the management of this difficult malignancy. We also review all original case reports and case series on parathyroid carcinoma, fulfilling the WHO criteria and with a follow-up of at least 6 months after initial parathyroidectomy, which were published in the English literature since the last large review on this topic in 2001 [3]. The literature search was conducted in PubMed using the keywords ("Parathyroid Neoplasms" OR "Parathyroid carcinoma" OR "Parathyroid cancer" OR "Parathyroid cancers") AND ("Case Reports" OR "case report" OR "case" OR "cases"). The last search was conducted on June 2, 2010. Patients with changes suggestive of carcinoma in the context of autonomous (tertiary) hyperparathyroidism were excluded because of the controversy in diagnosing malignancy in this case. Of an initial 872 publications, only 45 fulfilled the WHO criteria for parathyroid carcinoma and follow-up as per our search criteria, and were selected for further analysis.

\section{Case Report}

A 29-year-old man presented to his primary care physician with a 2-month history of severe weight loss $(20 \mathrm{~kg})$ associated with polyuria, polydipsia, nausea, muscle weakness, difficulty in concentrating and debilitating tiredness. On physical examination, he was clinically dehydrated and had a palpable hard, fixed swelling in the left side of the neck. He was using no medication and had no significant past or family history. Laboratory investigations revealed a severe hypercalcaemia of $4.9 \mathrm{mmol} / 1$ (normal range 2.15 $2.55 \mathrm{mmol} / \mathrm{l}$ ), a decreased creatinine clearance of $45 \mathrm{ml} / \mathrm{min}$ (normal $>120 \mathrm{ml} / \mathrm{min}$ ) and a 10 -fold increased serum intact parathyroid hormone (PTH) concentration: $88 \mathrm{pmol} / 1$ (normal $<8 \mathrm{pmol} / \mathrm{l}$ ). These findings suggested a diagnosis of severe primary hyperparathyroidism probably due to parathyroid carcinoma. No further investigations were requested; the patient was intensively rehydrated and referred for parathyroidectomy. At neck exploration, an enlarged parathyroid gland $(3.5 \times 3 \times 3.5 \mathrm{~cm})$ invading the ipsilateral thyroid lobe was found and removed from the left side of the neck (Fig. 1). A left hemithyroidectomy was also performed. The diagnosis of parathyroid carcinoma was confirmed histologically by the finding of capsular and vascular invasion. Serum calcium and PTH concentrations normalised within a few days of surgery associated with a significant improvement in renal function. A second surgery was performed within 1 month of the first surgery to ensure complete removal of all malignant tissue and provide a negative surgical margin. The patient was started on a course of radiotherapy, which had to be discontinued before completion because of severe pharyngitis and oesophagitis.

A recurrence of hyperparathyroidism was documented on routine laboratory control 3 years after parathyroidectomy. This was mild, with a serum calcium concentration of $2.74 \mathrm{mmol} / \mathrm{l}$, a PTH concentration of $8.4 \mathrm{pmol} / \mathrm{l}$ and a stable creatinine clearance of $75 \mathrm{ml} / \mathrm{min}$. The patient was completely asymptomatic. Tc99m-MIBI-SPECT and ultrasonography identified a lesion in the left side of the neck, which was found to be a local recurrence of the primary tumour at extensive bilateral neck exploration. The recurrent tumour had invaded the oesophagus, part of which was excised and reconstructed using tissue from the sternocleidomastoid muscle. The edges of the excised specimen were tumour free and resected draining lymph nodes were clear of tumour tissue. DNA analysis of the resected recurrent tumour demonstrated a somatic HRPT2 mutation, c.165delC located on exon 2 [22]. Despite the radical nature of the surgery, mild hyperparathyroidism persisted postoperatively, suggesting the presence of residual probably

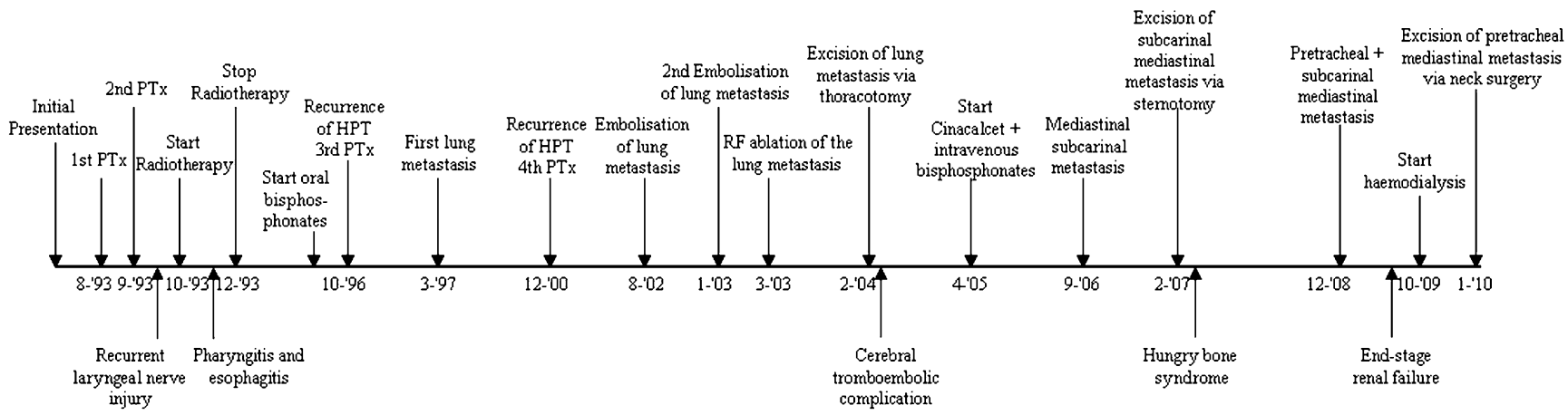

Fig. 1 Disease course of our patient spanning over 17 years 
metastatic tumour tissue. No further localisation studies or surgery were planned principally because of the patient's reluctance to undergo further surgery but also because of the mild nature of the hyperparathyroidism. Closer clinical monitoring was arranged.

Over the following 4 years, serum calcium and PTH concentrations and bone turnover markers slowly increased, and periods of dehydration were associated with transient worsening of renal function, which remained, however, on the whole stable despite persistent hypercalciuria and development of nephrocalcinosis. Progressive bone loss, predominantly cortical, was also documented and treatment with the non-nitrogen-containing bisphosphonate clodronate was started, resulting in normalisation of bone turnover and stabilisation of BMD measurements. Rehydration and treatment with bisphosphonates eventually failed in controlling hypercalcaemia and bone loss. Localisation studies were undertaken to identify the source of PTH production. Ultrasound (US), selective venous sampling for PTH (SVS) and CT scan of the neck succeeded in localising a small dense lesion in the left supraclavicular region. CT scan of the thorax also revealed a small solitary lesion of $1-\mathrm{cm}$ radius in the inferior lobe of the right lung (Fig. 2a). The patient refused to undergo a thoracotomy, but did agree to a fourth neck exploration at which a lymph node metastasis was excised from the left supraclavicular region (Fig. 1). Although less severe, hyperparathyroidism persisted postoperatively, as expected due to the unresected lung metastasis. Two attempts at embolisation of the lung metastasis failed to reduce tumour load. In contrast, radiofrequency ablation (RFA) of the lesion, although complicated by an episode of severe pleuritis, was very successful in achieving biochemical remission, with serum PTH concentrations decreasing from $265 \mathrm{pmol} / 1$ to $<1$ pmol/1 (Fig. 2), suggesting that the metastasis targeted with RFA was the only source of PTH secretion. However, remission was not permanent and $\mathrm{PTH}$ levels started to rise within 9 months of RFA. The patient finally agreed to undergo a right inferior lobectomy (Fig. 3), which was complicated by multiple small cerebral infarcts resulting in right haemianopia, dysphasia, acalculia and transient epilepsy. Disappointedly, there was also only partial improvement in serum calcium and PTH concentrations, which continued to increase within 2 months of lobectomy (Fig. 3).

Further localisation studies, in the form of Tc99mMIBI-SPECT and a CT scan of the neck and thorax, were unable to localise any residual pathological
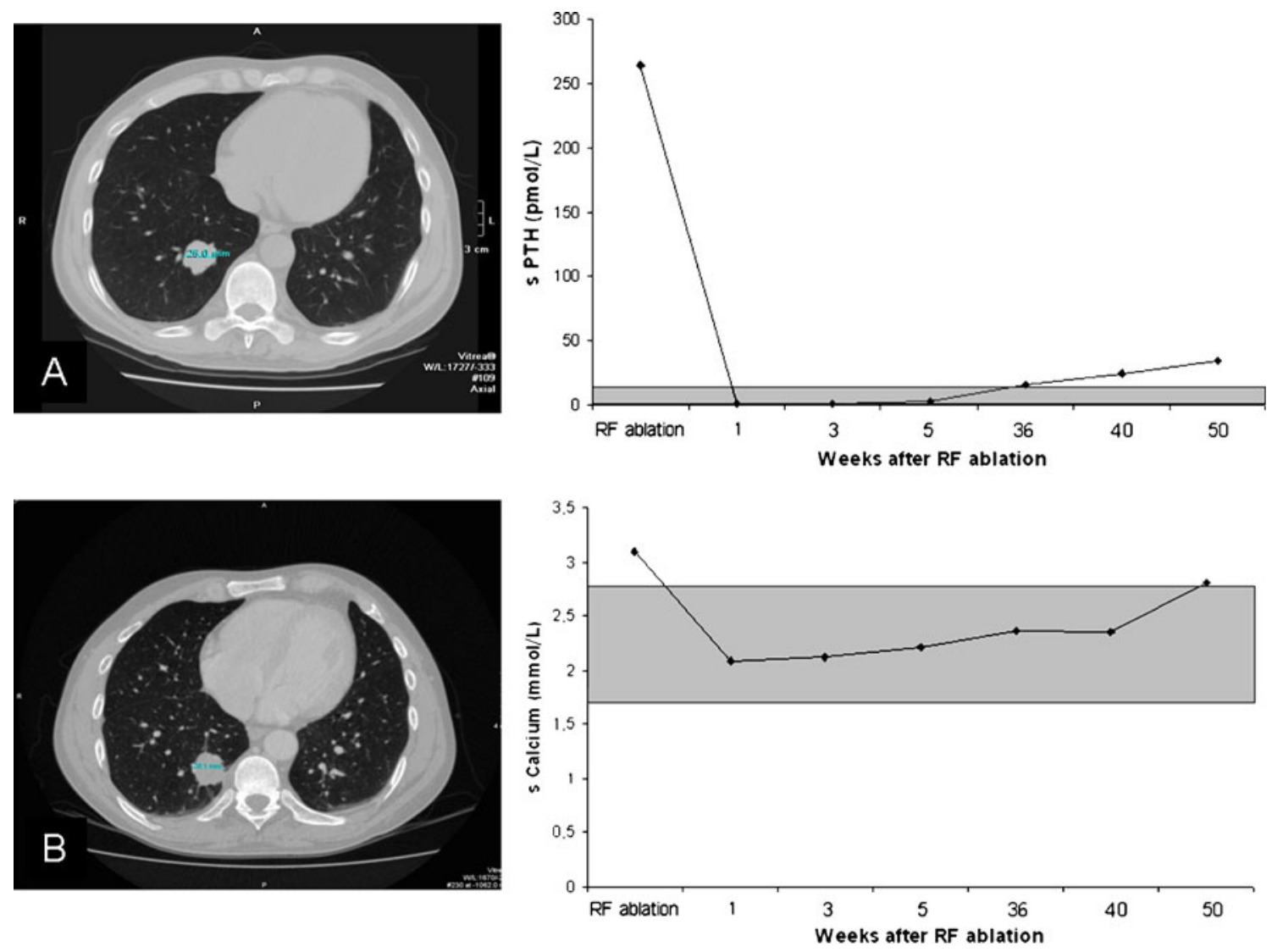

Fig. 2 Radiological (a pre-RF ablation, b after RF ablation) and biochemical changes after RF ablation of the lung metastasis, demonstrating a severe drop in serum PTH concentrations, which persisted for almost a year 

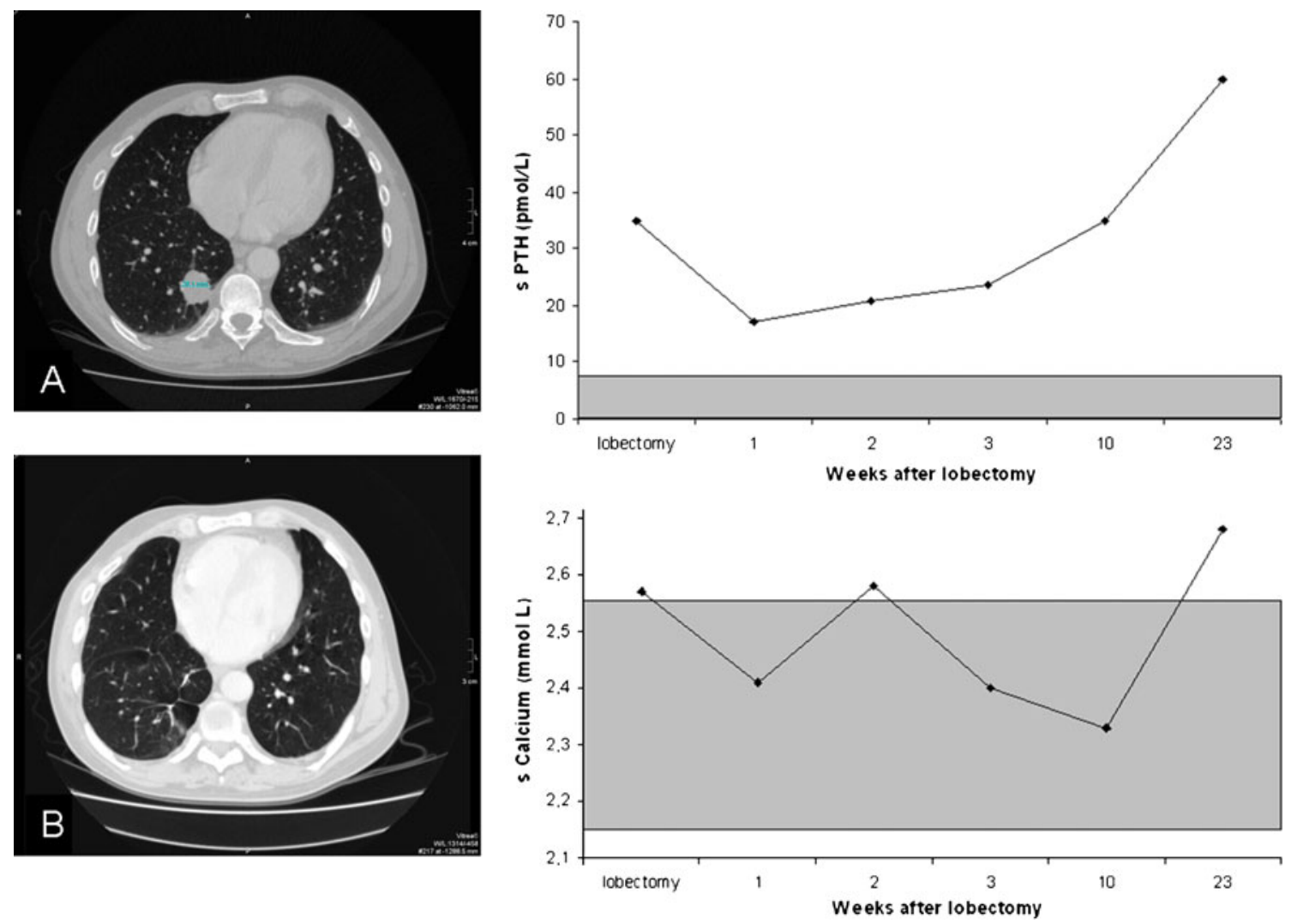

Fig. 3 Radiological and biochemical changes following lobectomy of the lung metastasis, which resulted in only partial improvement in serum calcium and PTH concentrations

parathyroid tissue suggesting the presence of (micro) metastasis. The now available calcimimetic cinacalcet was prescribed eventually at a maximum dose of $90 \mathrm{mg}$ twice daily, which decreased PTH concentrations from $120 \mathrm{pmol} / 1$ to $85 \mathrm{pmol} / \mathrm{l}$, stabilised calcium concentrations to about $2.80 \mathrm{mmol} / \mathrm{l}$ and stabilised creatinine clearance at $51 \mathrm{ml} /$ min. In the course of the following year, serum calcium and PTH concentrations further increased, however, despite maximal doses of cinacalcet and intermittent intravenous bisphosphonates.

Repeat Tc99m-MIBI-SPECT and CT scan of the thorax, now demonstrated a new large $(4 \times 3 \times 2 \mathrm{~cm})$ subcarinal mediastinal metastasis, which was successfully excised using a transpericardial approach (Fig. 4). The elimination of the source of PTH secretion was associated with a very severe hungry bone syndrome for which the patient needed intensive care for over 2 months, requiring very large doses of iv administered calcium (up to $14 \mathrm{~g} /$ day) and maximal doses of active vitamin $\mathrm{D}$ metabolites. Remission lasted for more than 1 year, after which PTH levels started to rise again and a new metastatic lesion was localised pretracheally in the upper mediastinum on CT scan of the thorax. There was also suggestion of a second subcarinal lesion at the site of the previously excised metastasis.
In view of the complications associated with his previous surgeries, the patient was at that stage again reluctant to undergo any further surgery. Within the next few months, PTH steadily increased associated with uncontrollable hypercalcaemia and deteriorating renal function eventually requiring haemodialysis using a very-low-dialysate calcium concentration. Localisation studies confirmed the two previously identified pretracheal and subcarinal mediastinal lesions. The pretracheal lesion was accessible from the neck and was radically removed by a cervical approach (Fig. 1). There were no post-operative complications and serum PTH concentration decreased from $590 \mathrm{pmol} / 1$ to $155 \mathrm{pmol} / \mathrm{l}$, as expected due to the remaining subcarinal mediastinal metastasis. Surgical removal of this metastasis would have required a third thoracotomy, which would be associated with too high an operative risk, especially in view of the patient's poor clinical condition. Excision of the metastasis by mediastinoscopy was also not considered to be an option because of multiple scarring due to previous surgeries. The patient remains on haemodialysis and is still treated with cinacalcet, with intermittent courses of intravenous bisphosphonates. The patient was not considered to be a candidate for PTH immunisation [23, 24] because of surgical clearance of cervical and mediastinal lymph nodes, poor general immune status and recurring infections. 
Fig. $4 \mathrm{CT}$ scan of the thorax demonstrating a large $(4 \times 3 \times$ $2 \mathrm{~cm}$ ) subcarinal mediastinal lymph node metastasis (white arrow) $(\mathbf{a}, \mathbf{b})$, which was successfully excised using a transpericardial approach $(\mathbf{c})$
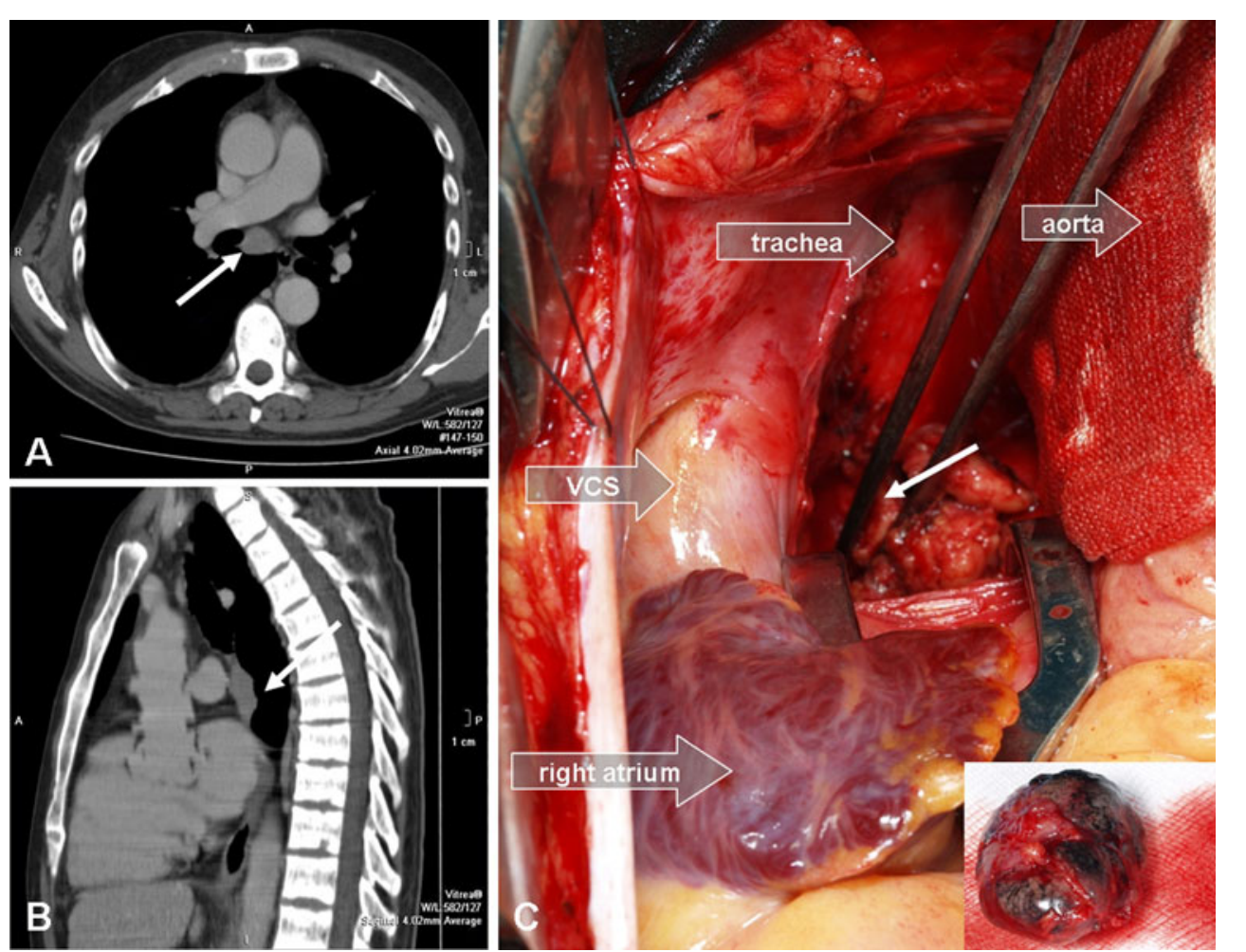

\section{Review of the Literature 2001-2010}

Sixty-two new cases have been reported in the literature since the last published review in 2001 [3], and data of these cases are summarised in Table 1. The median age of the reported patients was 50 years (21-79) and there was an equal number of men and women reported. Data on initial presentation were available in 43 of the 62 patients $(69 \%)$, 37 of which were symptomatic (86\%). Polyuria and polydipsia were reported in nine patients $(21 \%)$, constipation in seven $(16 \%)$, tiredness in $13(30 \%)$, bone and joint pain in $17(40 \%)$, muscle weakness in seven (16\%), nausea and vomiting in 10 $(23 \%)$ and weight loss in seven patients $(16 \%)$. Seventeen patients $(40 \%)$ had renal stones and six patients (14\%) had sustained a documented fracture. A palpable neck mass was reported in 22 of 27 patients in whom this was checked (81\%).

Similar to the review of 2001, surgery is still the most frequently chosen therapy for the initial treatment of parathyroid carcinoma but also for the treatment of recurrent or metastatic parathyroid carcinoma (Table 1). It is of note that the use of both chemotherapy and radiotherapy has decreased over the last 10 years, likely due to several reports and reviews which showed disappointing results [2-5]. The last case report on the use of chemotherapy and radiotherapy dates from 2005 and 2008, respectively (Table 1 ). In contrast to the review of 2001, reports on the use of the newer techniques embolisation $(n=1)$, radiofrequency ablation $(n=3)$ and PTH immunisation $(n=1)$ have been recently published, although the number of cases reported is still very limited. Use of the calcimimetic, cinacalcet, has increased over the last few years after the first report in 2007, while the use of bisphosphonates has remained stable.

The median follow-up reported in the 62 cases is relatively short at 3 years (range $0.5-20$ years), considering that the average time to a first recurrence is also approximately 3 years $[1,3]$. This could explain why only 27 of the 62 patients $(44 \%)$ developed a local recurrence and/or distant metastasis and nine patients $(15 \%)$ died as a result of parathyroid carcinoma during the reported followup period.

\section{Discussion}

To our knowledge, we report here one of the longest survival and follow-up in one centre of a patient with recurrent metastatic parathyroid carcinoma. Although parathyroid carcinoma is a slow-growing tumour, it is associated with a relatively high incidence of local recurrence $(40-82 \%$ within 5 years) $[1-3,5,6,13]$. Metastases tend to occur later in the course of the disease with spread to cervical nodes (30\%), lung (40\%) and liver $(10 \%)$, via both lymphatic and haematogenous routes [3]. Survival is estimated to range from $35 \%$ to $70 \%$ at 10 years $[1-6,13,14]$, with an average survival of 6 years after local recurrence, and of 4.5 years after the development of distant metastases [2]. 
In patients with parathyroid carcinoma, determinants of survival include delay in diagnosis, the presence of locally invasive features, distant metastasis at diagnosis and the radical nature of initial surgery $[3,6,13,14]$. A further important determinant of survival and disease-free survival is the due care taken at initial surgery to avoid rupturing the capsule of the gland to prevent local seeding of tumour tissue [3]. Initial surgery has been reported not to be radical in up to $48 \%$ of patients because of failure to establish the diagnosis of parathyroid carcinoma pre- or intra-operatively $[6,14]$. This was also the case in our patient at initial surgery, despite very suggestive clinical features of parathyroid carcinoma, in the form of severe hyperparathyroidism in a young male patient, palpable neck swelling and intra-operative features of local invasion. A second surgery had to be performed within 1 month of the first to ensure complete removal of all malignant tissue and to provide a negative surgical margin.

The main determinant of survival in all patients remains, however, the eventual inability to control hypercalcaemia $[2,5]$ and its association with fatal renal and cardiovascular complications [1, 2, 5, 7]. End-stage renal failure has been reported in up to $84 \%$ of patients due the deleterious effects of severe persistent hypercalcaemia on kidney function [2, 5, 7]. A persistently elevated circulating PTH level is also associated with increased bone turnover and bone loss predominantly at cortical sites and with increased risk of fractures. In parathyroid carcinoma, the main goal of treatment is control of hyperparathyroidism by eradicating the source of PTH secretion [7, 16].

Although the morbidity associated with re-exploration of the neck is estimated to be 6-17\% [7], that of re-exploration of the mediastinum or lungs is clearly much higher. In our patient, the recurrent laryngeal nerve had to be unavoidably sacrificed during the second surgical intervention due to invasive growth of remnants of the primary tumour, resulting in transient loss of voice and permanent hoarseness. Subsequent lung surgery was associated with severe thrombo-embolic complications.

The increasing morbidity attached to repeated surgical interventions has led to the search for other treatment options, such as radiotherapy, chemotherapy, RF ablation, embolisation, use of the calcimimetic cinacalcet and PTH immunisation. Although parathyroid tumours are relatively resistant to radiotherapy [2-5], this management approach showed some promise as adjuvant treatment for microscopic residual disease $[8,9]$. In our patient, the prescribed course of radiotherapy could not be completed due to the development of severe side effects in the form of pharyngitis and oesophagitis.

Embolisation of localised and/or ectopic parathyroid adenomas has been reported to have only limited success
[19, 25-27], and in metastatic parathyroid carcinoma, embolisation has only been described in combination with RF ablation in one patient [19]. In our patient, the outcome of twice attempted embolisation of a lung metastasis was disappointing, largely because it was not technically possible to selectively embolise the arterial branch that fed the lung metastasis.

The outcome of combined embolisation and RF ablation in a patient with liver metastasis due to parathyroid carcinoma [19], and the effectiveness of RF ablation in various types of cancer [28] has led us to consider RF ablation in our patient. This approach was indeed very successful in eradicating PTH production by the metastasis, as has also been subsequently reported in two patients with parathyroid carcinoma and lung metastases $[20,21]$. The beneficial effect was unfortunately transient, lasting only 9 months after the procedure, when PTH production was documented to resume from the same site.

In parathyroid carcinoma, attempts at reducing tumour load are not always successful, in which case control of serum calcium by other means becomes central to prevent the deleterious effects of hypercalcaemia on various organ systems. Intensive rehydration, use of medications such as bisphosphonates and calcimimetics and use of dialysis represent diverse means to control hypercalcaemia and are often used in combination. Bisphosphonates decrease the skeletal efflux of calcium from bone by suppressing osteoclast-mediated bone resorption and overall bone turnover [3]. These agents, however, have no effect on renal tubular reabsorption of calcium so that they improve hypercalcaemia but do not normalise serum calcium [3, 29]. Cinacalcet is a calcimimetic, which reduces parathyroid hormone secretion by binding to the calcium-sensing receptor on parathyroid cells, increasing the sensitivity of these cells to extracellular calcium concentrations [15, 30, 31]. These agents are widely used in the management of secondary and tertiary hyperparathyroidism in renal failure and have been also recently registered for the management of primary hyperparathyroidism, including that due to parathyroid carcinoma. In our patient, combined treatment with bisphosphonates and cinacalcet resulted in reasonable control of the hypercalcaemia initially, but eventually failed to do so, even when used at maximal doses. Dialysing against low-dialysate calcium became necessary in order to better control the hypercalcaemia.

The very high operative risks associated with a third thoracotomy, the patient's poor lung function and current metabolic status despite regular dialysis, preclude the option of further surgery to remove the identified source of PTH secretion. PTH immunisation is being explored as a potential non-invasive option, although experience with this approach is still limited and the patient's general condition may not permit its use $[23,24,32]$. 
Although we are rapidly running out of options in the management of our patient, we believe that we have nonetheless managed to secure for him a longer albeit not disease-free survival. This clinical case with a follow-up spanning over 17 years illustrates that the long-term management of patients with metastatic parathyroid carcinoma remains indeed a daunting task, despite all recent imaging, surgical and medical advances.

Declaration of Interest There is no conflict of interest that could be perceived as prejudicing the impartiality of the research reported.

Funding This research did not receive any specific grant from any funding agency in the public, commercial or not-for-profit sector.

Open Access This article is distributed under the terms of the Creative Commons Attribution Noncommercial License which permits any noncommercial use, distribution, and reproduction in any medium, provided the original author(s) and source are credited.

\section{References}

1. Kebebew E (2001) Parathyroid carcinoma. Curr Treat Options Oncol 2:347-354

2. Shane E, Bilezikian JP (1982) Parathyroid carcinoma: a review of 62 patients. Endocr Rev 3:218-226

3. Shane E (2001) Clinical review 122: parathyroid carcinoma. J Clin Endocrinol Metab 86:485-493

4. Schantz A, Castleman B (1973) Parathyroid carcinoma. A study of 70 cases. Cancer 31:600-605

5. Wynne AG, van Heerden J, Carney JA et al (1992) Parathyroid carcinoma: clinical and pathologic features in 43 patients. Medicine (Baltimore) 71:197-205

6. Hundahl SA, Fleming ID, Fremgen AM et al (1999) Two hundred eighty-six cases of parathyroid carcinoma treated in the U.S. between 1985-1995: a National Cancer Data Base Report. The American College of Surgeons Commission on Cancer and the American Cancer Society. Cancer 86:538-544

7. Kebebew E, Arici C, Duh QY et al (2001) Localization and reoperation results for persistent and recurrent parathyroid carcinoma. Arch Surg 136:878-885

8. Chow E, Tsang RW, Brierley JD et al (1998) Parathyroid carcinoma - the Princess Margaret Hospital experience. Int $\mathrm{J}$ Radiat Oncol Biol Phys 41:569-572

9. August DA, Flynn SD, Jones MA et al (1993) Parathyroid carcinoma: the relationship of nuclear DNA content to clinical outcome. Surgery 113:290-296

10. Tan MH, Morrison C, Wang P et al (2004) Loss of parafibromin immunoreactivity is a distinguishing feature of parathyroid carcinoma. Clin Cancer Res 10:6629-6637

11. Christmas TJ, Chapple CR, Noble JG et al (1988) Hyperparathyroidism after neck irradiation. Br J Surg 75:873-874

12. DeLellis RA (2006) World health organisation classification of tumours. Pathology and genetics of tumours of endocrine organs. AIRC, Lyon

13. Sandelin K, Auer G, Bondeson L et al (1992) Prognostic factors in parathyroid cancer: a review of 95 cases. World J Surg 16:724-731
14. Kleinpeter KP, Lovato JF, Clark PB et al (2005) Is parathyroid carcinoma indeed a lethal disease? Ann Surg Oncol 12:260-266

15. Szmuilowicz ED, Utiger RD (2006) A case of parathyroid carcinoma with hypercalcemia responsive to cinacalcet therapy. Nat Clin Pract Endocrinol Metab 2:291-296

16. Cheah WK, Rauff A, Lee KO et al (2005) Parathyroid carcinoma: a case series. Ann Acad Med Singapore 34:443-446

17. Bukowski RM, Sheeler L, Cunningham J et al (1984) Successful combination chemotherapy for metastatic parathyroid carcinoma. Arch Intern Med 144:399-400

18. Calandra DB, Chejfec G, Foy BK et al (1984) Parathyroid carcinoma: biochemical and pathologic response to DTIC. Surgery 96:1132-1137

19. Artinyan A, Guzman E, Maghami E et al (2008) Metastatic parathyroid carcinoma to the liver treated with radiofrequency ablation and transcatheter arterial embolization. J Clin Oncol 26:4039-4041

20. Iguchi T, Yasui K, Hiraki T et al (2008) Radiofrequency ablation of functioning lung metastases from parathyroid carcinoma. J Vasc Interv Radiol 19:462-464

21. Tochio M, Takaki H, Yamakado K et al (2010) A case report of 20 lung radiofrequency ablation sessions for 50 lung metastases from parathyroid carcinoma causing hyperparathyroidism. Cardiovasc Intervent Radiol 33:657-659

22. Haven CJ, van Puijenbroek M, Tan MH et al (2007) Identification of MEN1 and HRPT2 somatic mutations in paraffin-embedded (sporadic) parathyroid carcinomas. Clin Endocrinol (Oxf) 67:370-376

23. Betea D, Bradwell AR, Harvey TC et al (2004) Hormonal and biochemical normalization and tumor shrinkage induced by antiparathyroid hormone immunotherapy in a patient with metastatic parathyroid carcinoma. J Clin Endocrinol Metab 89:3413-3420

24. Bradwell AR, Harvey TC (1999) Control of hypercalcaemia of parathyroid carcinoma by immunisation. Lancet 353:370-373

25. Reidy JF, Ryan PJ, Fogelman I et al (1993) Ablation of mediastinal parathyroid adenomas by superselective embolization of the internal mammary artery with alcohol. Clin Radiol 47:170-173

26. Miller DL, Doppman JL, Chang R et al (1987) Angiographic ablation of parathyroid adenomas: lessons from a 10-year experience. Radiology 165:601-607

27. Doppman JL (1980) The treatment of hyperparathyroidism by transcatheter techniques. Cardiovasc Intervent Radiol 3:268-276

28. Hiraki T, Sakurai J, Tsuda T et al (2006) Risk factors for local progression after percutaneous radiofrequency ablation of lung tumors: evaluation based on a preliminary review of 342 tumors. Cancer 107:2873-2880

29. Hamdy NA, Gray RE, McCloskey E et al (1987) Clodronate in the medical management of hyperparathyroidism. Bone 8 (Suppl 1):S69-S77

30. Silverberg SJ, Rubin MR, Faiman C et al (2007) Cinacalcet hydrochloride reduces the serum calcium concentration in inoperable parathyroid carcinoma. J Clin Endocrinol Metab 92:3803-3808

31. Collins MT, Skarulis MC, Bilezikian JP et al (1998) Treatment of hypercalcemia secondary to parathyroid carcinoma with a novel calcimimetic agent. J Clin Endocrinol Metab 83:1083-1088

32. Horie I, Ando T, Inokuchi N et al (2010) First Japanese patient treated with parathyroid hormone peptide immunization for refractory hypercalcemia caused by metastatic parathyroid carcinoma. Endocr J 57:287-292

33. Savli H, Sevinc A, Sari R et al (2001) Occult parathyroid carcinoma in a patient with papillary thyroid carcinoma and Hashimoto's thyroiditis. J Endocrinol Invest 24:42-44

34. Tyler D III, Mandybur G, Dhillon G et al (2001) Intracranial metastatic parathyroid carcinoma: case report. Neurosurgery 48:937-939

35. Dotzenrath C, Goretzki PE, Sarbia M et al (2001) Parathyroid carcinoma: problems in diagnosis and the need for radical surgery even in recurrent disease. Eur J Surg Oncol 27:383-389 
36. Yamashita K, Suzuki S, Yumita W et al (2001) A case of familial isolated hyperparathyroidism with ectopic parathyroid cancer. Endocr J 48:453-458

37. Eurelings M, Frijns CJ, Jeurissen FJ (2002) Painful ophthalmoplegia from metastatic nonproducing parathyroid carcinoma: case study and review of the literature. Neuro Oncol 4:44-48

38. Brown JJ, Mohamed H, Williams-Smith L et al (2002) Primary hyperparathyroidism secondary to simultaneous bilateral parathyroid carcinoma. Ear Nose Throat J 81:395-1

39. Bhansali A, Kataria RN, Dutta P et al (2002) Parathyroid carcinoma: difficult management options. Indian J Cancer 39:119-122

40. Hundley JC, Albertson DA, Bradley RF et al (2003) Resection of pulmonary metastasis from parathyroid carcinoma. Am Surg 69:779-783

41. Schmidt JL, Perry RC, Philippsen LP et al (2002) Intrathyroidal parathyroid carcinoma presenting with only hypercalcemia. Otolaryngol Head Neck Surg 127:352-353

42. Schoretsanitis G, Melissas J, Kafousi M et al (2002) Synchronous parathyroid and papillary thyroid carcinoma: a case report. Am J Otolaryngol 23:382-385

43. Dionisi S, Minisola S, Pepe J et al (2002) Concurrent parathyroid adenomas and carcinoma in the setting of multiple endocrine neoplasia type 1: presentation as hypercalcemic crisis. Mayo Clin Proc 77:866-869

44. Rufener JB, Cohen JI (2003) Metachronous spread of parathyroid carcinoma to a retropharyngeal lymph node. Head Neck 25:968-971

45. Munson ND, Foote RL, Northcutt RC et al (2003) Parathyroid carcinoma: is there a role for adjuvant radiation therapy? Cancer 98:2378-2384

46. Dogan A, Algun E, Kisli E et al (2004) Calcaneal brown tumor with primary hyperparathyroidism caused by parathyroid carcinoma: an atypical localization. J Foot Ankle Surg 43:248-251

47. Kern M, Lee G, Robbins P et al (2004) Intracranial metastatic parathyroid carcinoma. Case report and review of the literature. J Neurosurg 101:1065-1069

48. Kirkby-Bott J, Lewis P, Harmer CL et al (2005) One stage treatment of parathyroid cancer. Eur J Surg Oncol 31:78-83

49. Lin SD, Tu ST, Hsu SR et al (2005) Synchronous parathyroid and papillary thyroid carcinoma. J Chin Med Assoc 68:87-91

50. Ahmad F, Strickland AD, Wright GM et al (2005) Laparoscopic microwave tissue ablation of hepatic metastasis from a parathyroid carcinoma. Eur J Surg Oncol 31:321-322

51. Chiofalo MG, Scognamiglio F, Losito $S$ et al (2005) Huge parathyroid carcinoma: clinical considerations and literature review. World J Surg Oncol 3:39

52. Yoshida S (2006) Intracranial metastatic parathyroid carcinoma: case report. Surg Neurol 65:81-83

53. Ikeda K, Tate G, Suzuki T et al (2006) Cytologic comparison of a primary parathyroid cancer and its metastatic lesions: a case report. Diagn Cytopathol 34:50-55

54. Pahlavan PS, Severin MC (2006) Parathyroid carcinoma: a rare case with mandibular brown tumor. Wien Klin Wochenschr 118:175-179

55. Agarwal G, Dhingra S, Mishra SK et al (2006) Implantation of parathyroid carcinoma along fine needle aspiration track. Langenbecks Arch Surg 391:623-626
56. Mezhir JJ, Melis M, Headley RC et al (2007) Successful palliation of hypercalcemia secondary to metastatic parathyroid cancer: an unusual indication for hepatic resection. J Hepatobiliary Pancreat Surg 14:410-413

57. Placzkowski K, Christian R, Chen H (2007) Radioguided parathyroidectomy for recurrent parathyroid cancer. Clin Nucl Med 32:358-360

58. Sahasranam P, Tran MT, Mohamed H et al (2007) Multiglandular parathyroid carcinoma: a case report and brief review. S Med J 100:841-844

59. Tkaczyk M, Czupryniak A, Nowicki M (2007) Ectopic mediastinal parathyroid carcinoma as a cause of dialysis-dependent renal failure. Hemodial Int 11:398-402

60. Tan GC, Shiran MS, Swaminathan M et al (2007) Large retrosternal parathyroid carcinoma with primary hyperparathyroidism. Asian J Surg 30:286-289

61. Iwata $\mathrm{T}$, Inoue $\mathrm{K}$, Morita $\mathrm{R}$ et al (2008) Functional large parathyroid carcinoma extending into the superior mediastinum. Ann Thorac Cardiovasc Surg 14:112-115

62. Montenegro FL, Chammas MC, Juliano AG et al (2008) Ethanol injection under ultrasound guidance to palliate unresectable parathyroid carcinoma. Arq Bras Endocrinol Metabol 52:707-711

63. Rathi MS, Ajjan R, Orme SM (2008) A case of parathyroid carcinoma with severe hungry bone syndrome and review of literature. Exp Clin Endocrinol Diab 116:487-490

64. Cetani F, Pardi E, Ambrogini E et al (2008) Hyperparathyroidism 2 gene (HRPT2, CDC73) and parafibromin studies in two patients with primary hyperparathyroidism and uncertain pathological assessment. J Endocrinol Invest 31:900-904

65. Temmim L, Sinowatz F, Hussein WI et al (2008) Intrathyroidal parathyroid carcinoma: a case report with clinical and histological findings. Diagn Pathol 3:46

66. Tamura G, Miyoshi H, Ogata SY et al (2009) Parathyroid carcinoma with anaplastic feature: association of a p53 gene mutation with anaplastic transformation. Pathol Int 59:107-110

67. Schoretsanitis G, Daskalakis M, Melissas J et al (2009) Parathyroid carcinoma: clinical presentation and management. Am J Otolaryngol $30: 277-280$

68. Kung B, Winokur R, Cognetti D et al (2009) Parathyroid carcinoma: a rare cause of primary hyperparathyroidism. Ear Nose Throat J 88:E10-E13

69. Marcy PY, Thariat J, Sudaka A et al (2009) Synchronous parathyroid and papillary thyroid carcinomas. Thyroid 19:1131-1133

70. Yuan SF, Yan W, Ji G et al (2010) Surgical therapy of bilateral parathyroid carcinoma: report of an unusual case. Eur J Surg Oncol 36:107-109

71. Rock K, Fattah N, O'Malley D et al (2010) The management of acute parathyroid crisis secondary to parathyroid carcinoma: a case report. J Med Case Reports 4:28

72. Yong TY, Li JY (2010) Mediastinal parathyroid carcinoma presenting with severe skeletal manifestations. J Bone Miner Metab 28:591-594

73. Chaychi L, Belbruno K, Golding A et al (2010) An unusual manifestation of parathyroid carcinoma in the setting of papillary thyroid cancer. Endocr Pract 16:664-668 